\title{
Depression, anxiety and associated factors among Chinese adolescents during the COVID-19 outbreak: a comparison of two cross-sectional studies
}

Xu Chen ${ }^{1}$, Han Qi ${ }^{1}$, Rui Liu' ${ }^{1,2,3,4}$, Yuan Feng ${ }^{1}$, Wen Li, ${ }^{2,3,4}$, Mi Xiang ${ }^{5}$, Teris Cheung ${ }^{6}$, Todd Jackson', Gang Wang ${ }^{1}$ and Yu-Tao Xiang ${ }^{2,3,4}$

\begin{abstract}
The 2019 coronavirus disease (COVID-19) is a public health emergency of international concern. In China, all schools were shut down and students were home quarantined to prevent disease spread; these steps could have potential negative effects on mental health of adolescents. This study aimed to examine changes in depression and anxiety among Chinese adolescents during the COVID-19 epidemic, and explore factors associated with depression and anxiety. Two survey administrations were conducted among Chinese adolescents between February 20 and February 27 and between April 11 and April 19, 2020, respectively. The Center for Epidemiological Studies-Depression Scale (CES-D) and the 7-item Generalized Anxiety Disorder (GAD-7) scale were used to assess depressive symptoms and anxiety symptoms, respectively. A total of 9554 and 3886 adolescents participated in the first and second surveys. During the initial survey, the prevalence of depression was 36.6\% (95\% Cl: 35.6-37.6\%) while the prevalence of anxiety was 19\% (95\% Cl: 18.2-19.8\%). Rates of depression and anxiety increased to 57.0\% (95\% Cl: $55.4-58.6 \%$ ) and 36.7\% (95\% Cl: 35.2-38.2\%), respectively, in the second survey. Multivariable logistic regression analyses revealed that group membership in the second survey, female gender, senior secondary school enrollment, and concerns about entering a higher grade were positively associated with both depression and anxiety. Conversely, a sleep duration of $\geq 6 \mathrm{~h} /$ day, an exercise duration $\geq 30 \mathrm{~min} /$ day, having the same as typical or higher study efficiency during the COVID-19 outbreak, and living in provinces with 1000-9999 confirmed COVID-19 cases were negatively associated with depression and anxiety. In conclusion, compared to figures reported during the COVID-19 outbreak, the prevalence of depression and anxiety in Chinese adolescents significantly increased after the initial outbreak. Regular screening and appropriate interventions are urgently needed to reduce the risk for emotional disturbances among adolescents during and after the initial COVID-19 outbreaks.
\end{abstract}

Correspondence: Gang Wang (gangwangdoc@ccmu.edu.cn) or Yu-Tao Xiang (xyutly@gmail.com)

${ }^{1}$ The National Clinical Research Center for Mental Disorders \& Beijing Key Laboratory of Mental Disorders, Beijing Anding Hospital \& the Advanced Innovation Center for Human Brain Protection, Capital Medical University, Beijing, China

${ }^{2}$ Unit of Psychiatry, Institute of Translational Medicine, Faculty of Health

Sciences, University of Macau, Macao SAR, China

Full list of author information is available at the end of the article

These authors contributed equally: Xu Chen, Han Qi, Rui Liu, Yuan Feng

\section{Introduction}

Coronavirus disease 2019 (COVID-19) was first reported in December 2019 in Wuhan, Hubei province, China ${ }^{1}$ and was subsequently reported in more than 200 countries and territories ${ }^{2}$. On January 30, 2020, the World Health Organization (WHO) declared COVID-19 to be a public health emergency of international concern ${ }^{3}$. Due to fear of infection, potentially fatal consequences of

\section{(c) The Author(s) 2021}

(c) Open Access This article is licensed under a Creative Commons Attribution 4.0 International License, which permits use, sharing, adaptation, distribution and reproduction cc) in any medium or format, as long as you give appropriate credit to the original author(s) and the source, provide a link to the Creative Commons license, and indicate if changes were made. The images or other third party material in this article are included in the article's Creative Commons license, unless indicated otherwise in a credit line to the material. If material is not included in the article's Creative Commons license and your intended use is not permitted by statutory regulation or exceeds the permitted use, you will need to obtain permission directly from the copyright holder. To view a copy of this license, visit http://creativecommons.org/licenses/by/4.0/. 
COVID-19 and related problems such as unemployment, financial strain, quarantine and long-term lockdown in some areas, mental health problems resulting from the COVID-19 outbreak are common in different subpopulations including confirmed patients ${ }^{4}$, frontline health professionals, and the elderly ${ }^{5-7}$. However, to date very few studies have focused on mental health of adolescents during the pandemic, although this subpopulation is also vulnerable to experiencing mental health problems $^{8,9}$.

To reduce rapid transmission, the Chinese Ministry of Education suggested postponement of the spring 2020 semester in primary and secondary schools on January 30, 2020. Consequently, all face-to face teaching in schools has been suspended in China during the COVID19 pandemic and students must receive education online at home ${ }^{10,11}$. As such, primary and secondary school students in China have stayed at home and have had to communicate with their classmates and teachers via the internet or other social media forums for more than 5 months. Although some schools in China were reopened after the initial COVID-19 outbreak was well controlled in April, $2020^{12}$, many schools remain closed due to the fear of a second outbreak. Prolonged home stay is associated with a range of negative outcomes for adolescents, such as decreased social interaction with peers, reduced physical activity, increased conflicts with parents, and academic pressures due to sudden changes of traditional learning methods ${ }^{13-15}$. Presumably, all of these changes could increase risk for mental health problems, particularly depression and anxiety. For example, a recent cross-sectional study has found the prevalence of depression and anxiety to be $43.7 \%$ and $37.4 \%$, respectively, among Chinese adolescents during the COVID-19 outbreak ${ }^{16}$. However, to date, comparisons of the mental health status of adolescents have not been made between different stages of the COVID-19 outbreak. Thus, we set out to compare the prevalence of depression and anxiety among adolescents at different stage of COVID-19 outbreak as well as correlates of these experiences. Considering that the impact of persistent financial crisis on parental behaviors ${ }^{8,17}$, potential intensification of social isolation and loneliness ${ }^{18}$, and cumulative pressure of long-term online learning due to the COVID-19 outbreak could increase the risk of mental health problems among adolescents, we hypothesized that the prevalence of depression and anxiety would increase among Chinese adolescents over time.

\section{Methods}

\section{Settings and participants}

In this national study, two administrations of a mental health survey were conducted through the collaborative research network of the National Clinical Research Center for Mental Disorders, China; all 34 provinces of China were included. The first survey was administered from February 20 to 27 (during the COVID-19 outbreak) while the second round was administered from April 11 to 19 (after the COVID-19 outbreak). Due to risk of infection, traditional face-to-face interviews were not adopted. Following other studies ${ }^{19-21}$, snowball sampling was used, and data were collected using a smart phone-based WeChat-Wenjuanxing application. The application has more than 1 billion users in China and has been widely used for student management in most secondary schools in China during the COVID-19 outbreak. Inclusion criteria included: (1) secondary school students aged between 11 and 20 years; and (2) living in China during the COVID-19 outbreak. Participants with pre-existing major psychiatric disorders (e.g., psychotic disorder, major depression, and autism) were excluded based on participant self-reports. All participants were required to provide written informed consent. For participants under 18 years old, guardians were required to provide written consent. This study was approved by the Medical Ethical Committee of Beijing Anding Hospital, Capital Medical University, China.

\section{Data Collection}

A pre-designed data collection form was used to collect socio-demographic data and information about clinical characteristics. Study sites were classified according to the total number of COVID-19 patients at provincial level based on a report of the National Health Commission of China (http://www.nhc.gov.cn) released on February 27, 2020.

Depression was assessed with the Chinese version of the Center for Epidemiological Studies-Depression Scale $(C E S-D)^{22}$. The CES-D is a 20-item self-report questionnaire ${ }^{23}$. Each item scored from 0 (not at all) to 3 (a lot). CES-D total scores of $>15$ were considered "having depression" ${ }^{24}$. The Chinese version of CES-D has been validated in adolescents with satisfactory psychometric properties $^{25}$. Anxiety symptoms were measured using the 7-item self-report Generalized Anxiety Disorder Scale (GAD-7). Each item scored from 0 (not at all) to 3 (nearly every day $)^{26}$, with total scores ranging from 0 to 21 . Cutoff values of 5,10, 15 indicate mild, moderate, and severe anxiety, respectively ${ }^{27}$. In this study, participants who scored $>4$ on the GAD-7 were classified as having anxiety. The Chinese version of GAD-7 has satisfactory psychometric properties ${ }^{28}$.

\section{Statistical analysis}

All analyses were performed with SPSS version 21.0 software. A subset of adolescents who completed the first survey administration also participated in the second administration. Therefore, to avoid any overlap 
between the two study samples, those who participated in both surveys were excluded from the second sample. Mann-Whitney $U$-tests were used to compare CES-D and GAD-7 total scores between the two surveys. Demographic correlates of depression and anxiety during each assessment were assessed using chi-square tests. Multivariable logistic regression analyses with "enter" method were performed to examine correlates of depression and anxiety, respectively. In these analyses, either depression or anxiety was entered as the dependent variable while significant correlates based on univariate analyses were independent variables. A $P$ value of $<0.05$ level was considered statistically significant (two-tailed).

\section{Results}

\section{Demographic characteristics for the first and second survey}

Of 9744 adolescents who enrolled in the initial survey, 9554 (47.9\% male) completed the assessment and their responses were included in analyses. In addition, of 10,706 adolescents who volunteered for the second survey administration, $10,605(46.2 \%$ male $)$ completed the assessment. After excluding students who had also completed the initial survey $(n=6719)$, the final sample included for analyses from the second survey comprised 3886 students. Using power analysis ${ }^{29}$, the achieved power of the two surveys in this study was greater than 0.9, indicating that the sample size was large enough. Demographic characteristics based on presence/absence of depression and anxiety were summarized in Table 1.

\section{Depression and anxiety in the first and second survey}

Mean CES-D and GAD-7 total scores were compared between the first and second surveys in Table 1. The mean CES-D total score for the whole sample was $14.06 \pm$ 10.80 in the first survey and $19.29 \pm 11.82$ in the second survey $(Z=-24.78, P<0.001)$. Mean GAD-7 total score also increased from $2.28 \pm 3.54$ in the first survey to $3.98 \pm 4.46$ in the second survey $(Z=-24.88, P<0.001)$. Except for students in vocational schools and those living in provinces with 10-99 confirmed COVID-19 cases, mean CES-D and GAD-7 total scores based on demographic characteristics and study-related variables were also significantly higher in the second survey than the first survey (Table 1).

Prevalence estimates of depression (CES-D $>15)$ and anxiety (GAD-7 $>4$ ) were also compared between the surveys. In the first survey, the prevalence of depression was $36.6 \%$ (95\% CI: 35.6-37.6\%) while the rate of anxiety was $19 \%$ (95\% CI: $18.2-19.8 \%$ ). In the second survey, corresponding figures were $57.0 \%$ (95\% CI: 55.4-58.6\%) for depression and 36.7\% (95\% CI: 35.2-38.2\%) for anxiety. Table 2 breaks down depression and anxiety prevalence stratified by demographic characteristics and study-related variables.

\section{Correlates of depression and anxiety}

Multivariable logistic regression analyses revealed that increased risk of depression was associated with completing the second survey ( $\mathrm{OR}=1.47,95 \% \mathrm{CI}: 1.34-1.61)$, female gender $(\mathrm{OR}=1.21,95 \% \mathrm{CI}$ : $1.12-1.31)$, senior secondary school $(\mathrm{OR}=1.41,95 \% \mathrm{CI}: 1.29-1.54)$, vocational schools $(\mathrm{OR}=1.25,95 \% \mathrm{CI}: 1.04-1.51)$, and being concerned about entering a higher grade $(\mathrm{OR}=1.75,95 \%$ CI: 1.62-1.89). Furthermore, significant correlates of lower risk of depression included sleep duration $6-8 \mathrm{~h} /$ day $(\mathrm{OR}=0.45,95 \% \mathrm{CI}$ : $0.37-0.53)$ and $>8 \mathrm{~h} /$ day $(\mathrm{OR}=$ 0.32 , 95\% CI: $0.27-0.38)$, exercise durations of $30-60 \mathrm{~min} /$ day $(\mathrm{OR}=0.64,95 \%$ CI: $0.60-0.70)$ and $\geq 60 \mathrm{~min} /$ day $(\mathrm{OR}=$ $0.67,95 \%$ CI: $0.59-0.77)$, study duration $4-8 \mathrm{~h} /$ day $(\mathrm{OR}=0.87,95 \% \mathrm{CI}: 0.78-0.98)$, having higher than typical study efficiency ( $\mathrm{OR}=0.64,95 \% \mathrm{CI}: 0.56-0.74$ ), participating in distant learning ( $\mathrm{OR}=0.68,95 \% \mathrm{CI}$ : 0.56-0.81), and living in provinces with number of confirmed cases 1000-9999 (OR =0.47, 95\% CI: 0.24-0.92) (Table 3).

Similarly, increased risk of anxiety was related to completing the second survey ( $\mathrm{OR}=1.64,95 \% \mathrm{CI}: 1.48-1.81)$, female gender $(\mathrm{OR}=1.23,95 \% \mathrm{CI}: 1.13-1.34)$, senior secondary school $(\mathrm{OR}=1.27,95 \% \mathrm{CI}$ : $1.15-1.41)$, and being concerned about entering a higher grade $(\mathrm{OR}=$ 1.74, 95\% CI: 1.59-1.91). Lower risk of anxiety was independently associated with studying in ordinary schools $(\mathrm{OR}=0.78,95 \% \mathrm{CI}$ : $0.66-0.92)$, sleep duration $6-8 \mathrm{~h} /$ day $(\mathrm{OR}=0.47,95 \% \mathrm{CI}: 0.40-0.55)$ and $>8 \mathrm{~h} /$ day (OR $=0.34,95 \% \mathrm{CI}: 0.28-0.40)$, exercise durations of $30-60 \mathrm{~min} /$ day $(\mathrm{OR}=0.73,95 \% \mathrm{CI}: \quad 0.67-0.80)$ and $\geq 60 \mathrm{~min} /$ day $\quad(\mathrm{OR}=0.84,95 \% \mathrm{CI}: 0.73-0.98)$, having higher than typical study efficiency $(\mathrm{OR}=0.81,95 \% \mathrm{CI}$ : $0.69-0.96)$, and living in provinces with number of confirmed cases $>100(\mathrm{OR}=0.41,95 \% \mathrm{CI}: 0.22-0.80)$ (Table 3).

\section{Discussion}

This was the first study to compare rates of depression and anxiety among adolescents during versus after the initial COVID-19 outbreak. Compared with the corresponding figures during the initial COVID-19 outbreak in China, the prevalence of depression and anxiety significantly increased after the initial COVID-19 outbreak had remitted.

Compared to the prevalence of depression (24.3\%; $95 \%$ CI: $21.3-27.6 \%)^{30}$ and anxiety (14.1\%; $95 \%$ CI: $13.2-15.0 \%)^{31}$ among Chinese adolescents prior to the COVID-19 outbreak, both depression (36.6\%; 95\% CI: $35.6-37.6 \%)$ and anxiety (19\%; 95\% CI: $18.2-19.8 \%)$ were more common during the COVID-19 outbreak. These increases could be due to unpleasant experiences brought 
Table 1 The comparison of CES-D and GAD-7 scores between the first $(n=9554)$ and second $(n=3886)$ surveys.

\begin{tabular}{|c|c|c|c|c|c|c|c|c|}
\hline \multirow[t]{2}{*}{ Variables } & \multicolumn{4}{|l|}{ Scores of CES-D } & \multicolumn{4}{|l|}{ Scores of GAD-7 } \\
\hline & The first survey & The second survey & $z$ & $P$ value & The first survey & The second survey & $z$ & $P$ value \\
\hline \multicolumn{9}{|l|}{ Gender } \\
\hline male & $13.17 \pm 10.13$ & $18.74 \pm 11.66$ & -18.09 & $<0.001$ & $1.98 \pm 3.37$ & $3.77 \pm 4.49$ & -17.47 & $<0.001$ \\
\hline female & $14.87 \pm 11.33$ & $19.75 \pm 11.93$ & -16.79 & $<0.001$ & $2.55 \pm 3.66$ & $4.16 \pm 4.43$ & -17.43 & $<0.001$ \\
\hline \multicolumn{9}{|l|}{ Age (years) } \\
\hline $11-15$ & $12.67 \pm 10.27$ & $17.77 \pm 11.96$ & -15.43 & $<0.001$ & $1.95 \pm 3.28$ & $3.58 \pm 4.52$ & -14.12 & $<0.001$ \\
\hline $16-20$ & $16.03 \pm 11.23$ & $20.18 \pm 11.65$ & -14.50 & $<0.001$ & $2.75 \pm 3.83$ & $4.22 \pm 4.41$ & -16.22 & $<0.001$ \\
\hline \multicolumn{9}{|l|}{ Grade } \\
\hline Junior secondary school & $12.66 \pm 10.31$ & $17.69 \pm 12.12$ & -14.25 & $<0.001$ & $1.94 \pm 3.29$ & $3.56 \pm 4.62$ & -12.62 & $<0.001$ \\
\hline Senior secondary school & $15.91 \pm 11.16$ & $20.06 \pm 11.60$ & -14.95 & $<0.001$ & $2.73 \pm 3.79$ & $4.19 \pm 4.37$ & -16.60 & $<0.001$ \\
\hline \multicolumn{9}{|l|}{ School type } \\
\hline Provincial key schools & $13.95 \pm 10.87$ & $19.99 \pm 11.97$ & -7.12 & $<0.001$ & $2.64 \pm 4.09$ & $4.77 \pm 4.46$ & -8.78 & $<0.001$ \\
\hline Municipal key schools & $13.01 \pm 10.58$ & $19.08 \pm 12.67$ & -9.06 & $<0.001$ & $2.37 \pm 3.64$ & $4.28 \pm 4.99$ & -8.35 & $<0.001$ \\
\hline Ordinary schools & $13.75 \pm 10.69$ & $19.52 \pm 11.77$ & -22.90 & $<0.001$ & $2.16 \pm 3.43$ & $3.97 \pm 4.41$ & -22.33 & $<0.001$ \\
\hline Vocational schools & $15.86 \pm 11.09$ & $16.10 \pm 10.40$ & -0.77 & 0.441 & $2.43 \pm 3.53$ & $2.86 \pm 4.02$ & -1.18 & 0.237 \\
\hline Major class & $13.12 \pm 10.70$ & $20.55 \pm 11.52$ & -18.20 & $<0.001$ & $2.38 \pm 3.70$ & $4.27 \pm 4.32$ & -15.92 & $<0.001$ \\
\hline \multicolumn{9}{|l|}{ Sleep duration/day } \\
\hline$<6 \mathrm{~h}$ & $22.06 \pm 13.50$ & $26.30 \pm 13.28$ & -4.79 & $<0.001$ & $4.94 \pm 5.65$ & $6.67 \pm 6.01$ & -4.95 & $<0.001$ \\
\hline $6-8 \mathrm{~h}$ & $14.56 \pm 10.70$ & $19.11 \pm 11.32$ & -18.30 & $<0.001$ & $2.39 \pm 3.45$ & $3.84 \pm 4.13$ & -18.52 & $<0.001$ \\
\hline$>8 \mathrm{~h}$ & $12.38 \pm 10.06$ & $15.37 \pm 10.89$ & -6.89 & 0.019 & $1.81 \pm 3.15$ & $2.83 \pm 3.90$ & -6.90 & $<0.001$ \\
\hline \multicolumn{9}{|l|}{ Study duration/day } \\
\hline$<4 \mathrm{~h}$ & $16.66 \pm 11.49$ & $17.97 \pm 11.57$ & -2.06 & 0.039 & $2.68 \pm 4.00$ & $3.63 \pm 4.54$ & -3.80 & $<0.001$ \\
\hline $4-8 \mathrm{~h}$ & $13.30 \pm 10.19$ & $18.46 \pm 11.38$ & -19.29 & $<0.001$ & $2.08 \pm 3.24$ & $3.64 \pm 4.17$ & -18.57 & $<0.001$ \\
\hline$>8 \mathrm{~h}$ & $13.98 \pm 11.22$ & $21.53 \pm 12.50$ & -17.84 & $<0.001$ & $2.40 \pm 3.72$ & $4.80 \pm 4.87$ & -17.13 & $<0.001$ \\
\hline \multicolumn{9}{|l|}{ Exercise duration/day } \\
\hline$<30 \min$ & $16.13 \pm 11.31$ & $21.65 \pm 12.08$ & -17.22 & $<0.001$ & $2.68 \pm 3.82$ & $4.55 \pm 4.67$ & -17.82 & $<0.001$ \\
\hline $30-60 \mathrm{~min}$ & $12.29 \pm 9.90$ & $16.89 \pm 10.73$ & -15.89 & $<0.001$ & $1.91 \pm 3.15$ & $3.35 \pm 3.97$ & -15.52 & $<0.001$ \\
\hline$>60 \mathrm{~min}$ & $12.31 \pm 10.57$ & $17.82 \pm 12.55$ & -7.93 & $<0.001$ & $2.10 \pm 3.62$ & $3.87 \pm 4.88$ & -7.42 & $<0.001$ \\
\hline \multicolumn{9}{|l|}{ Change of study efficiency } \\
\hline Lower than before & $17.25 \pm 11.63$ & $20.67 \pm 11.94$ & -11.38 & $<0.001$ & $3.03 \pm 4.10$ & $4.33 \pm 4.53$ & -13.68 & $<0.001$ \\
\hline About the same & $12.60 \pm 9.97$ & $16.40 \pm 10.98$ & -11.14 & $<0.001$ & $1.87 \pm 3.10$ & $3.20 \pm 4.09$ & -10.73 & $<0.001$ \\
\hline Higher than before & $12.18 \pm 10.39$ & $17.26 \pm 11.62$ & -6.32 & $<0.001$ & $2.19 \pm 3.53$ & $3.70 \pm 4.82$ & -4.86 & $<0.001$ \\
\hline \multicolumn{9}{|c|}{ No. of COVID-19 cases at provincial level } \\
\hline $10-99$ & $14.07 \pm 11.16$ & $24.17 \pm 13.54$ & -1.19 & 0.235 & $2.70 \pm 4.03$ & $2.50 \pm 3.27$ & -0.77 & 0.442 \\
\hline 100-999 & $14.29 \pm 10.61$ & $19.49 \pm 11.86$ & -22.89 & $<0.001$ & $2.22 \pm 3.38$ & $4.08 \pm 4.50$ & -23.23 & $<0.001$ \\
\hline 1000-9999 & $13.08 \pm 10.86$ & $17.39 \pm 11.63$ & -5.74 & $<0.001$ & $1.94 \pm 3.32$ & $2.83 \pm 3.97$ & -3.91 & $<0.001$ \\
\hline$>10,000$ & $14.59 \pm 11.17$ & $16.63 \pm 10.28$ & -2.46 & 0.014 & $2.41 \pm 3.66$ & $3.16 \pm 3.51$ & -2.75 & 0.006 \\
\hline Participating in distance learning & $13.80 \pm 10.73$ & $19.29 \pm 11.81$ & -25.57 & $<0.001$ & $2.24 \pm 3.52$ & $3.97 \pm 4.44$ & -24.96 & $<0.001$ \\
\hline $\begin{array}{l}\text { Concerned about entering a } \\
\text { higher grade }\end{array}$ & $16.32 \pm 11.29$ & $20.70 \pm 11.80$ & -16.16 & $<0.001$ & $2.83 \pm 3.95$ & $4.39 \pm 4.55$ & -17.64 & $<0.001$ \\
\hline
\end{tabular}

Bolded Values: $P<0.05$.

The scores of CES-D and GAD-7 were presented as mean \pm standard deviation.

CES-D Center for Epidemiological Studies-Depression Scale, GAD-7 7-item Generalized Anxiety Disorder Scale.

by the COVID-19 outbreak such as fear of infection, limited public services, frustration, loneliness or lack of interpersonal contact with peers, and limited personal space at home.

Significant increases in prevalence of depression and anxiety among adolescents in the second survey may be attributed to the several factors. First, although timely online mental health education and services have been established during the COVID-19 outbreak in China ${ }^{6,32}$, their content is not designed for special populations such as adolescents. On a related note, most secondary school students were highly occupied with heavy study burdens that might interfere with involvement and benefits of these mental health services. Second, COVID-19 was well controlled in China during the second survey but most students suffering from depressive and/or anxiety symptoms during the first survey did not receive timely effective treatments due to inaccessible mental health services in many areas; as such, symptoms may not have remitted. Third, due to school closures and social distancing, 
Table 2 The comparison of the prevalence of depression and anxiety between the first and second surveys.

\begin{tabular}{|c|c|c|c|c|c|c|c|c|}
\hline \multirow[t]{2}{*}{ Variables } & \multicolumn{4}{|c|}{ Depression (CES-D > 15) $n=5713$} & \multicolumn{4}{|c|}{ Anxiety (GAD-7 > 4) $n=3240$} \\
\hline & The first survey & The second survey & $x^{2}$ & $P$ value & The first survey & The second survey & $X^{2}$ & $P$ value \\
\hline \multicolumn{9}{|l|}{ Gender } \\
\hline male & $1514(33.1)$ & $962(54.8)$ & 252.31 & $<0.001$ & $759(16.6)$ & $614(35.0)$ & 253.41 & $<0.001$ \\
\hline female & $1984(39.9)$ & $1253(58.8)$ & 215.16 & $<0.001$ & $1,055(21.2)$ & $812(38.1)$ & 219.85 & $<0.001$ \\
\hline \multicolumn{9}{|l|}{ Age (years) } \\
\hline $11-15$ & $1745(31.1)$ & $721(50.6)$ & 188.68 & $<0.001$ & $872(15.6)$ & $465(32.6)$ & 214.82 & $<0.001$ \\
\hline $16-20$ & $1753(44.4)$ & $1494(60.7)$ & 161.45 & $<0.001$ & $942(23.9)$ & $961(39.1)$ & 167.66 & $<0.001$ \\
\hline \multicolumn{9}{|l|}{ Grade } \\
\hline Junior secondary school & $1693(31.0)$ & $617(48.9)$ & 145.24 & $<0.001$ & $848(15.5)$ & $414(32.8)$ & 200.48 & $<0.001$ \\
\hline Senior secondary school & $1805(44.1)$ & $1598(60.9)$ & 181.04 & $<0.001$ & $966(23.6)$ & $1,012(38.6)$ & 172.70 & $<0.001$ \\
\hline \multicolumn{9}{|l|}{ School type } \\
\hline Provincial key schools & $223(36.9)$ & $158(58.7)$ & 36.01 & $<0.001$ & $138(22.8)$ & $124(46.1)$ & 47.89 & $<0.001$ \\
\hline Municipal key schools & $537(32.4)$ & $204(55.4)$ & 68.68 & $<0.001$ & $319(19.3)$ & $133(36.1)$ & 49.45 & $<0.001$ \\
\hline Ordinary schools & 1891 (35.1) & $1742(58.1)$ & 413.57 & $<0.001$ & 947 (17.6) & 1103 (36.8) & 383.93 & $<0.001$ \\
\hline Vocational schools & 847 (44.4) & $111(44.6)$ & 0.003 & 0.956 & $410(21.5)$ & $66(26.5)$ & 3.224 & 0.073 \\
\hline Major class & 799 (32.7) & $657(63.7)$ & 285.62 & $<0.001$ & $493(20.2)$ & $417(40.4)$ & 153.56 & $<0.001$ \\
\hline \multicolumn{9}{|l|}{ Sleep duration/day } \\
\hline$<6 h$ & $283(63.5)$ & $322(75.9)$ & 16.01 & $<0.001$ & $185(41.5)$ & $233(55.0)$ & 15.81 & $<0.001$ \\
\hline $6-8 \mathrm{~h}$ & 2089 (39.0) & $1626(57.3)$ & 252.07 & $<0.001$ & $1085(20.2)$ & $1029(36.3)$ & 249.18 & $<0.001$ \\
\hline$>8 \mathrm{~h}$ & $1126(30.0)$ & 267 (42.7) & 39.35 & $<0.001$ & $544(14.5)$ & $164(26.2)$ & 54.02 & $<0.001$ \\
\hline \multicolumn{9}{|l|}{ Study duration/day } \\
\hline$<4 h$ & $704(45.7)$ & $163(50.9)$ & 2.97 & 0.085 & $341(22.1)$ & $105(32.8)$ & 16.65 & $<0.001$ \\
\hline $4-8 h$ & $1711(34.3)$ & $1298(54.4)$ & 270.35 & $<0.001$ & $862(17.3)$ & 796 (33.3) & 239.77 & $<0.001$ \\
\hline$>8 \mathrm{~h}$ & 1083 (35.9) & $695(64.8)$ & 269.47 & $<0.001$ & $611(20.3)$ & $479(44.7)$ & 241.47 & $<0.001$ \\
\hline \multicolumn{9}{|l|}{ Exercise duration/day } \\
\hline$<30$ min & $1924(43.8)$ & $1.232(65.5)$ & 247.84 & $<0.001$ & $999(22.7)$ & $799(42.5)$ & 250.76 & $<0.001$ \\
\hline $30-60 \mathrm{~min}$ & $1289(30.3)$ & $777(48.9)$ & 174.43 & $<0.001$ & $651(15.3)$ & $492(31.0)$ & 179.82 & $<0.001$ \\
\hline$>60 \mathrm{~min}$ & $285(31.3)$ & $206(49.5)$ & 40.92 & $<0.001$ & $164(18.0)$ & $135(32.5)$ & 34.29 & $<0.001$ \\
\hline \multicolumn{9}{|l|}{ Change of study efficiency } \\
\hline Lower than before & $1449(47.1)$ & $1605(61.8)$ & 122.36 & $<0.001$ & $791(25.7)$ & $1032(39.7)$ & 126.97 & $<0.001$ \\
\hline About the same & $1751(31.7)$ & $514(47.0)$ & 94.83 & $<0.001$ & $849(15.4)$ & $332(30.4)$ & 139.76 & $<0.001$ \\
\hline Higher than before & $298(31.1)$ & $96(49.0)$ & 23.21 & $<0.001$ & $174(18.1)$ & $62(31.6)$ & 18.21 & $<0.001$ \\
\hline \multicolumn{9}{|c|}{ No. of COVID-19 cases at provincial level } \\
\hline 10-99 & $21(58.3)$ & $4(66.7)$ & 0.15 & 0.700 & $17(47.2)$ & $2(33.3)$ & 0.40 & 0.527 \\
\hline 100-999 & 2721 (37.2) & 2067 (58.2) & 424.92 & 0.002 & 1415 (19.4) & $1332(37.5)$ & 415.41 & $<0.001$ \\
\hline 1000-9999 & $560(33.1)$ & $91(44.2)$ & 9.92 & $<0.001$ & $277(16.4)$ & $53(25.7)$ & 11.14 & 0.001 \\
\hline$>10,000$ & 196 (37.5) & $53(43.8)$ & 1.62 & 0.203 & $105(20.1)$ & $39(32.2)$ & 8.30 & 0.004 \\
\hline Participating in distance learning & $3181(35.5)$ & $2156(57.0)$ & 504.96 & $<0.001$ & $1672(18.7)$ & $1380(36.5)$ & 463.79 & $<0.001$ \\
\hline $\begin{array}{l}\text { Concerned about entering a } \\
\text { higher grade }\end{array}$ & $1955(44.9)$ & $1789(62.4)$ & 213.09 & $<0.001$ & $1052(24.1)$ & $1183(41.3)$ & 237.32 & $<0.001$ \\
\hline
\end{tabular}

Bolded Values: $P<0.05$.

The percentages of CES-D $>15$ and GAD-7 $>4$ were presented as $n(\%)$.

CES-D Center for Epidemiological Studies-Depression Scale, GAD-7 7-item Generalized Anxiety Disorder Scale.

adolescents may have experienced losses of close peer relationships, which could increase risk for depression and anxiety ${ }^{18}$. Furthermore, lack of outdoor exercise ${ }^{33,34}$ and prolonged use of the Internet and smart phones at home $^{35,36}$ could increase risk for these disturbances. Fourth, in the second survey administration (i.e., after the initial COVID-19 outbreak), $88.3 \%$ of students still had not returned to school, although most of their parents had returned to work according to the relevant regulations in China. Lack of clarity about returning to school during this semester as well as lack of care and supervision from parents could have contributed to further increases in the risk for mental health problems. Finally, final examinations that are traditionally held in May-June were approaching yet face-to-face teaching could not be offered to many students. This combination may have increased the likelihood of mental health problems.

Consistent with previous findings ${ }^{16,37}$, female adolescents and those in senior secondary school were more likely to have anxiety and depression. We also found 
Table 3 Independent correlates of depression and anxiety of the whole sample $(n=13,440)$.

\begin{tabular}{|c|c|c|c|c|c|c|c|c|}
\hline \multirow[t]{3}{*}{ Variables } & \multicolumn{4}{|c|}{ Depression } & \multicolumn{4}{|l|}{ Anxiety } \\
\hline & \multirow[t]{2}{*}{$P$ value } & \multirow[t]{2}{*}{$O R$} & \multicolumn{2}{|c|}{$95 \% \mathrm{Cl}$ for OR } & \multirow[t]{2}{*}{$P$ value } & \multirow[t]{2}{*}{$O R$} & \multicolumn{2}{|c|}{$95 \% \mathrm{Cl}$ for OR } \\
\hline & & & Lower & Upper & & & Lower & Upper \\
\hline Second survey completion & $<0.001$ & 1.47 & 1.34 & 1.61 & $<0.001$ & 1.64 & 1.48 & 1.81 \\
\hline Female & $<0.001$ & 1.21 & 1.12 & 1.31 & $<0.001$ & 1.23 & 1.13 & 1.34 \\
\hline Senior secondary school & $<0.001$ & 1.41 & 1.29 & 1.54 & $<0.001$ & 1.27 & 1.15 & 1.41 \\
\hline \multicolumn{9}{|l|}{ School type } \\
\hline Provincial key schools & Ref & & & & Ref & & & \\
\hline Municipal key schools & 0.551 & 0.95 & 0.80 & 1.13 & 0.064 & 0.84 & 0.69 & 1.01 \\
\hline Ordinary schools & 0.336 & 1.08 & 0.92 & 1.26 & 0.003 & 0.78 & 0.66 & 0.92 \\
\hline Vocational schools & 0.015 & 1.25 & 1.04 & 1.51 & 0.327 & 0.90 & 0.74 & 1.11 \\
\hline Major class & 0.050 & 0.92 & 0.84 & 1.00 & 0.265 & 1.06 & 0.96 & 1.17 \\
\hline \multicolumn{9}{|l|}{ Sleep duration/day } \\
\hline$<6 \mathrm{~h}$ & Ref & & & & Ref & & & \\
\hline $6-8 h$ & $<0.001$ & 0.45 & 0.37 & 0.53 & $<0.001$ & 0.47 & 0.40 & 0.55 \\
\hline$>8 \mathrm{~h}$ & $<0.001$ & 0.32 & 0.27 & 0.38 & $<0.001$ & 0.34 & 0.28 & 0.40 \\
\hline \multicolumn{9}{|l|}{ Study duration/day } \\
\hline$<4 \mathrm{~h}$ & Ref & & & & Ref & & & \\
\hline $4-8 h$ & 0.023 & 0.87 & 0.78 & 0.98 & 0.098 & 0.89 & 0.78 & 1.02 \\
\hline$>8 \mathrm{~h}$ & 0.758 & 1.02 & 0.90 & 1.16 & 0.096 & 1.13 & 0.98 & 1.31 \\
\hline \multicolumn{9}{|l|}{ Exercise duration/day } \\
\hline$<30$ min & Ref & & & & Ref & & & \\
\hline $30-60 \mathrm{~min}$ & $<0.001$ & 0.64 & 0.60 & 0.70 & $<0.001$ & 0.73 & 0.67 & 0.80 \\
\hline$>60 \min$ & $<0.001$ & 0.67 & 0.59 & 0.77 & 0.025 & 0.84 & 0.73 & 0.98 \\
\hline \multicolumn{9}{|l|}{ Change of study efficiency } \\
\hline Lower than before & Ref & & & & Ref & & & \\
\hline About the same & $<0.001$ & 0.65 & 0.60 & 0.71 & $<0.001$ & 0.72 & 0.65 & 0.79 \\
\hline Higher than before & $<0.001$ & 0.64 & 0.56 & 0.74 & 0.014 & 0.81 & 0.69 & 0.96 \\
\hline \multicolumn{9}{|l|}{ No. of COVID-19 cases at provincial level } \\
\hline $10-99$ & Ref & & & & Ref & & & \\
\hline 100-999 & 0.159 & 0.62 & 0.32 & 1.21 & 0.008 & 0.41 & 0.22 & 0.80 \\
\hline 1000-9999 & 0.028 & 0.47 & 0.24 & 0.92 & 0.001 & 0.31 & 0.16 & 0.60 \\
\hline$>10,000$ & 0.135 & 0.59 & 0.30 & 1.18 & 0.013 & 0.42 & 0.21 & 0.83 \\
\hline Participating in distance learning & $<0.001$ & 0.68 & 0.56 & 0.81 & 0.637 & 0.95 & 0.77 & 1.18 \\
\hline Concerned about entering a higher grade & $<0.001$ & 1.75 & 1.62 & 1.89 & $<0.001$ & 1.74 & 1.59 & 1.91 \\
\hline
\end{tabular}

Due to Collinearity between age and grade, age was not entered in the multivariable logistic regression analysis. Bolded values: $P<0.05$.

Cl confidential Interval, $O R$ odds ratio.

students who reported daily study durations of more than $8 \mathrm{~h}$ and concerns about entering a higher grade were more likely to report depression and anxiety. Long hours of study are often associated with heavy homework demands, insufficient sleep, and overuse of computers, all of which could have a negative impact on adolescent 
mental health ${ }^{20,38,39}$. In China, secondary school students in higher grades often face higher academic stress levels ${ }^{40,41}$ because they need to prepare for college entrance examinations that have significant implications for future education and occupation opportunities ${ }^{41,42}$. Because many students could not receive traditional teaching due to the COVID-19 outbreak, they may have been more likely to suffer from depression and anxiety.

In this study, adolescents whose sleep durations were more than $6 \mathrm{~h} /$ day, whose exercise durations were more than $30 \mathrm{~min} /$ day, and whose study efficiency was higher than the level perceived before the COVID-19 outbreak were at significantly lower risk of depression and anxiety, results that echo previous findings ${ }^{43,44}$. Additionally, adolescents enrolled in ordinary schools were less likely to have anxiety compared to those in key schools where higher levels of academic stress are expected due to higher teacher and parent performance expectations and increased peer competition ${ }^{40,45,46}$. Adolescents participating in distance learning had a lower risk of depression, which may be attributed, in part, to more frequent communication and support from teachers and classmates ${ }^{47}$, online communication experiences that can reduce negative feelings of adolescents ${ }^{48,49}$. Finally, to control the spread of infection, provinces with more confirmed COVID-19 cases usually have more strict quarantine measures as well as timely mental health preventive measures and education. Aside from improving residents' knowledge about COVID-19, these resources could enhance their sense of control and confidence in battling the COVID-19 outbreak, in turn, reducing the negative impact of higher COVID-19 rates on their mental health ${ }^{5,20}$. In line with these contentions, this study confirmed that adolescents living in provinces with more than 100 confirmed COVID-19 cases had a lower risk of anxiety.

Strengths of this study included its two-round survey and the large sample size. Several limitations should also be noted. First, some potentially important correlates of depression or anxiety such as social support and physical health, could not be examined for logistical reasons (e.g., absence of resources to perform physical exams). Second, causal relationships between mental health problems and related variables could not be examined due to the crosssectional design and absence of experimental manipulations. Third, standardized diagnostic instruments such as the Structured Clinical Interview for the DSM-IV (SCID) could not be administered due to safety requirements associated with minimizing face-to-face contacts during the outbreak. Finally, because students with pre-existing major psychiatric disorders were excluded, findings may not generalize to this group.

In conclusion, depression and anxiety were common among adolescents in China during the initial COVID-19 outbreak and their prevalence increased significantly $\sim 6$ weeks later even though the outbreak was more under control. Although psychological interventions such as hotlines and online psychological counseling services, had been provided for the general public, students may have been less able to directly benefit from the generic content of such resources. Therefore, considering the negative impact of depression and anxiety on daily life and health outcomes, timely screening and appropriate interventions, such as online psychological counseling tailored for concerns specific to adolescents, are urgently needed to reduce the likelihood of emotional disturbances among adolescents during and after the initial COVID-19 outbreaks.

\section{Acknowledgements}

The study was supported by the National Key R\&D Program of China (2017YFC1311100); the National Science and Technology Major Project for investigational new drug (2018ZX09201-014); the Beijing Municipal Science \& Technology Commission (Z181100001518005, D171100007017001); and the National Natural Science Foundation of China (81901368).

\section{Author details \\ ${ }^{1}$ The National Clinical Research Center for Mental Disorders \& Beijing Key Laboratory of Mental Disorders, Beijing Anding Hospital \& the Advanced Innovation Center for Human Brain Protection, Capital Medical University, Beijing, China. ${ }^{2}$ Unit of Psychiatry, Institute of Translational Medicine, Faculty of Health Sciences, University of Macau, Macao SAR, China. ${ }^{3}$ Center for Cognition and Brain Sciences, University of Macau, Macao SAR, China. Institute of Advanced Studies in Humanities and Social Sciences, University of Macau, Macao SAR, China. ${ }^{5}$ School of Public Health, Shanghai Jiao Tong University, Shanghai, China. ${ }^{6}$ School of Nursing, Hong Kong Polytechnic University, Hong Kong SAR, China. ${ }^{7}$ Department of Psychology, University of Macau, Macao SAR, China}

\section{Author contributions}

Study design: X.C., H.Q., G.W., and Y.T.X. Data collection, analysis, and interpretation: X.C., H.Q., R.L., Y.F., and W.L. Drafting of the manuscript: H.Q., R.L., M.X., and Y.T.X. Critical revision of the manuscript: T.C. and T.J. Approval of the final version for publication: all co-authors.

\section{Conflict of interest}

The authors declare no competing interests.

\section{Publisher's note}

Springer Nature remains neutral with regard to jurisdictional claims in published maps and institutional affiliations.

Received: 4 September 2020 Revised: 14 January 2021 Accepted: 28 January 2021

Published online: 02 March 2021

\section{References}

1. Zhu, N. et al. A novel coronavirus from patients with Pneumonia in China, 2019. N. Engl. J. Med 382, 727-733 (2020).

2. World Health Organization. The Coronavirus disease (COVID-19) outbreak. https://www.who.int (2020).

3. World Health Organization. COVID-19 Public Health Emergency of International Concern (PHEIC) Global research and innovation forum. https://www. who.int/publications/m/item/covid-19-public-health-emergency-ofinternational-concern-(pheic)-global-research-and-innovation-forum (2020).

4. Gu, Y., Zhu, Y., Xu, F., Xi, J. \& Xu, G. Factors associated with mental health outcomes among patients with COVID-19 treated in the Fangcang shelter hospital in China. Asia Pac. Psychiatry, e12443 (2020). 
5. Xiang, Y. T. et al. Timely mental health care for the 2019 novel coronavirus outbreak is urgently needed. Lancet Psychiatry 7, 228-229 (2020).

6. Liu, S. et al. Online mental health services in China during the COVID-19 outbreak. Lancet Psychiatry 7, e17-e18 (2020).

7. Venugopal, V. C., Mohan, A. \& Chennabasappa, L. K. Status of mental health and its associated factors among the general populace of India during COVID19 pandemic. Asia Pac. Psychiatry, e12412 (2020).

8. Fegert, J. M., Vitiello, B., Plener, P. L. \& Clemens, V. Challenges and burden of the Coronavirus 2019 (COVID-19) pandemic for child and adolescent mental health: a narrative review to highlight clinical and research needs in the acute phase and the long return to normality. Child Adolesc. Psychiatry Ment. Health 14, 1-11 (2020)

9. World Health Organization. Adolescent mental health. https://www.who.int/ news-room/fact-sheets/detail/adolescent-mental-health (2019).

10. Xinhuanet. China Focus: Schools start online courses as epidemic control postpones new semester. http://www.xinhuanet.com/english/2020-02/17/ c_138792006.htm (2020).

11. Xinhuanet. China postpones school semester amid novel coronavirus outbreak. http://www.xinhuanet.com/english/2020-01/28/c_138738646.htm (2020).

12. Xinhuanet. China says its COVID-19 peak is over. http://www.xinhuanet.com/ english/2020-03/12/c_138870547.htm (2020).

13. Zhu, S. et al. The immediate mental health impacts of the COVID-19 pandemic among people with or without quarantine managements. Brain Behav. Immun. 87, 56-58 (2020).

14. Brooks, S. K. et al. The psychological impact of quarantine and how to reduce it: rapid review of the evidence. Lancet 395, 912-920 (2020).

15. Kılınçel, Ş., Kılınçel, O., Muratdağı, G., Aydın, A. \& Usta, M. B. Factors affecting the anxiety levels of adolescents in home-quarantine during COVID-19 pandemic in Turkey. Asia Pac. Psychiatry, e12406 (2020).

16. Zhou, S. J. et al. Prevalence and socio-demographic correlates of psychological health problems in Chinese adolescents during the outbreak of COVID-19. Eur. Child Adolesc. Psychiatry 29, 749-758 (2020).

17. Lee, D., Brooks-Gunn, J., McLanahan, S. S., Notterman, D. \& Garfinkel, I. The Great Recession, genetic sensitivity, and maternal harsh parenting. Proc. Natl Acad. Sci. USA 110, 13780-13784 (2013).

18. Loades, M. E. et al. Rapid systematic review: the impact of social isolation and loneliness on the mental health of children and adolescents in the context of COVID-19. J. Am. Acad. Child Adolesc. Psychiatry 59, 1218-1239 e1213. (2020).

19. Zhang, W. R. et al. Mental health and psychosocial problems of medical health workers during the COVID-19 epidemic in China. Psychother. Psychosom. 89, 242-250 (2020).

20. Zhong, B. L. et al. Knowledge, attitudes, and practices towards COVID-19 among Chinese residents during the rapid rise period of the COVID-19 outbreak: a quick online cross-sectional survey. Int. J. Biol. Sci. 16, 1745-1752 (2020).

21. Guo, Y. et al. Mental health disorders and associated risk factors in quarantined adults during the COVID-19 ouotbreak in China: cross-sectional study. J. Med. Internet Res. 22, e20328 (2020).

22. Radloff, L. The CES-D scale: a self-report depression scale for research in the general population. Appl. Psychol. Meas. 385-401 (1977).

23. Chen, Z. Y., Yang, X. D. \& Li, X. Y. Psychometric features of CES-D in Chinese adolescents (in Chinese). Chin. J. Clin. Psychol. 17, 443-445 (2009). +448.

24. Zhang, J. et al. Development of the Chinese age norms of CES-D in urban area (in Chinese). Chin. Ment. Health J. 24, 139-143 (2010).

25. Sun, X. Y., Li, Y. X., Yu, C. Q. \& Li, L. M. Reliability and validity of depression scales of Chinese version: a systematic review (in Chinese). J. Chin. Epidemiol. 38, 110-116 (2017).

26. Spitzer, R. L., Kroenke, K., Williams, J. B. \& Lowe, B. A brief measure for assessing generalized anxiety disorder: the GAD-7. Arch. Intern. Med. 166, 1092-1097 (2006).
27. Liu, Y., Zhang, W. B. \& Cai, J. Relation of anxiety and depression to lifestyle in junior high school students (in Chinese). Chin. Ment. Health J. 31, 235-240 (2017).

28. He, X., Li, C., Qian, J., Cui, H. S. \& Wu, W. Reliability and validity of a generalized anxiety scale in general hospital outpatients. Shanghai Arch. Psychiatry 22, 200-203 (2010).

29. Cohen, J. Statistical Power Analysis for the Behavioral Sciences 2nd edn (Academic press, 1988).

30. Tang, X., Tang, S., Ren, Z. \& Wong, D. F. K. Prevalence of depressive symptoms among adolescents in secondary school in mainland China: a systematic review and meta-analysis. J. Affect. Disord. 245, 498-507 (2019).

31. Jin, Y. et al. Prevalence and risk factors of anxiety status among students aged 13-26 years. Int. J. Clin. Exp. Med 7, 4420-4426 (2014).

32. Wang, D. et al. Clinical characteristics of 138 hospitalized patients with 2019 novel coronavirus-infected Pneumonia in Wuhan, China. JAMA 323, 1061-1069 (2020).

33. Wegner, M. et al. Systematic review of meta-analyses: exercise effects on depression in children and adolescents. Front. Psychiatry 11, 81 (2020).

34. Yin, X. Q. et al. The promotive effects of peer support and active coping on the relationship between bullying victimization and depression among chinese boarding students. Psychiatry Res. 256, 59-65 (2017).

35. Woods, H. C. \& Scott, H. \#Sleepyteens: social media use in adolescence is associated with poor sleep quality, anxiety, depression and low self-esteem. J. Adolesc. 51, 41-49 (2016).

36. Gao, J. et al. Mental health problems and social media exposure during COVID-19 outbreak. PLOS ONE 15, e0231924 (2020).

37. Fausiah, F., Turnip, S. S. \& Hauff, E. Gender differences and the correlates of violent behaviors among high school students in a post-conflict area in Indonesia. Asia Pac. Psychiatry 12, e12383 (2020)

38. Boers, E., Afzali, M. H., Newton, N. \& Conrod, P. Association of screen time and depression in adolescence. JAMA Pediatrics 173, 853-859 (2019).

39. Stiglic, N. \& Viner, R. M. Effects of screentime on the health and well-being of children and adolescents: a systematic review of reviews. BMJ Open $\mathbf{9}$, e023191 (2019).

40. Zhao, X., Selman, R. L. \& Haste, H. Academic stress in Chinese schools and a proposed preventive intervention program. Cogent Educ. 2, 1000477 (2015).

41. Tang, X., Tang, S., Ren, Z. \& Wong, D. F. K. Psychosocial risk factors associated with depressive symptoms among adolescents in secondary schools in mainland china: a systematic review and meta-analysis. J. Affect. Disord. 263, 155-165 (2020)

42. Li, H. \& Zhang, W. Peer support: easing up the relationship between adolescents' learning pressure and depression. Chin. J. Spec. Educ. 10, 87-91 (2014).

43. Wang, G., Zhang, Y., Zhao, J., Zhang, J. \& Jiang, F. Mitigate the effects of home confinement on children during the COVID-19 outbreak. Lancet 395, 945-947 (2020).

44. World Health Organization. Mental health and psychosocial considerations during the COVID-19 outbreak. https://www.who.int/publications/i/item/ WHO-2019-nCoV-MentalHealth-2020.1 (2020).

45. Xiao, J. Academic Stress, Test Anxiety, and Performance in a Chinese High School Sample: The Moderating Effects of Coping Strategies and Perceived Social Support. Doctor of Philosophy thesis, Georgia State Univ. (2013).

46. Sun, J., Dunne, M. P., Hou, X. Y. \& Xu, A. Q. Educational stress among Chinese adolescents: individual, family, school and peer influences. Educ. Rev. $\mathbf{6 5}$, 284-302 (2013)

47. Ren, P., Qin, X., Zhang, Y. \& Zhang, R. Is social support a cause or consequence of depression? A longitudinal study of adolescents. Front. Psychol. 9, 1634-1634 (2018).

48. Knowles, M. L., Haycock, N. \& Shaikh, I. Does Facebook magnify or mitigate threats to belonging? Soc. Psychol. 46, 313-324 (2015).

49. Orben, A., Tomova, L. \& Blakemore, S. J. The effects of social deprivation on adolescent development and mental health. Lancet Child Adolesc. Health 4 , 634-640 (2020). 